\title{
Early DEtection of wEaring off in Parkinson disease: The DEEP study
}

\author{
F. Stocchi ${ }^{\mathrm{a}, *}$, A. Antonini ${ }^{\mathrm{b}}$, P. Barone ${ }^{\mathrm{c}}$, M. Tinazzi $^{\mathrm{d}}$, M. Zappia ${ }^{\mathrm{e}}$, M. Onofrj ${ }^{\mathrm{f}}$, S. Ruggieri ${ }^{\mathrm{g}}$, \\ L. Morgante ${ }^{\mathrm{h}}$, U. Bonuccelli ${ }^{\mathrm{i}}$, L. Lopiano ${ }^{\mathrm{j}}$, P. Pramstaller ${ }^{\mathrm{k}}$, A. Albanese ${ }^{1}$, M. Attar ${ }^{\mathrm{m}}$, \\ V. Posocco ${ }^{\mathrm{m}}, \mathrm{D}$. Colombo ${ }^{\mathrm{m}}, \mathrm{G}$. Abbruzzese ${ }^{\mathrm{n}}$, on behalf of DEEP study group
}

a Department of Neurology, Institute of Research and Medical Care, IRCCS San Raffaele, Rome, Italy

${ }^{\mathrm{b}}$ Department for Parkinson's disease, IRCCS San Camillo, Venice, Italy

'School of Medicine, University of Salerno, Salerno, Italy

${ }^{\mathrm{d}}$ U.O. Neurologia Ospedale Civile Maggiore, Borgo Trento, Verona, Italy

e Clinica Neurologica I Policlinico Universitario, Catania, Italy

${ }^{\mathrm{f}}$ CeSI-Centro Studi Invecchiamento Fondazione Università Gabriele D’Annunzio, Chieti, Italy

${ }^{g}$ Neurologia Istituto Mediterraneo Neuromed, Isernia, Italy

h Dipartimento di Neuroscienze Università di Messina, Messina, Italy

${ }^{\mathrm{i}}$ U.O. Neurologia Ospedale Versilia, Lucca, Italy

${ }^{\mathrm{j}}$ Neurologia 4 A.S.O. Molinette, Turin, Italy

${ }^{\mathrm{k}}$ Neurologia Ospedale di Bolzano, Bolzano, Italy

${ }^{1}$ Neurologia I Istituto Nazionale Neurologico C. Besta, Università Cattolica del Sacro Cuore, Milan, Italy

${ }^{\mathrm{m}}$ Novartis Pharma Italia, Medical Department, Origgio, Italy

${ }^{\mathrm{n}}$ Department of Neurosciences, University of Genoa, Genoa, Italy

\section{A R T I C L E I N F O}

\section{Article history:}

Received 12 March 2013

Received in revised form

23 October 2013

Accepted 25 October 2013

\section{Keywords:}

Parkinson's disease

Wearing-off

Wearing-off questionnaire

Quality of life

\begin{abstract}
A B S T R A C T
Objective: Assessing the frequency of Wearing-Off (WO) in Parkinson's disease (PD) patients, and its impact on Quality of Life (QoL).

Methods: Consecutive ambulatory patients, who were on dopaminergic treatment for $\geq 1$ year, were included in this multicentre, observational cross-sectional study. In a single visit, WO was diagnosed based on neurologist assessment as well as using the validated Italian version of a patient self-rated 19question Wearing-Off Questionnaire (WOQ-19); WO was defined for scores $\geq 2$. QoL was evaluated by the 8-item Parkinson's Disease Questionnaire (PDQ-8).

Results: 617 subjects were included, with a mean anti-Parkinson treatment duration of $6.6 \pm 4.6$ years, $87.2 \%$ were on levodopa treatment. Neurologists identified presence of WO in 351 subjects (56.9\%), whereas 415 subjects $(67.3 \%$ ) were identified by the self-administered WOQ-19. In patients with a $<2.5$ years disease duration, WO was diagnosed in 12 subjects (21.8\%) by neurologists and in 23 subjects (41.8\%) by the WOQ- 19 . The most frequent WO symptoms, as identified by WOQ-19, were "slowness of movements" (55.8\%) and "reduced dexterity" (48.8\%). Younger age, female gender, Unified Parkinson's Disease Rating Scale (UPDRS) part II score and duration of anti-Parkinson treatment were found significantly associated with WO. The number of motor $(p<0.0001)$ and non-motor $(p<0.0001)$ WO symptoms correlated with PDQ-8 total score. Conclusions: WO is common already at the early stages of PD and is underestimated by routine neurological clinical evaluation. The number of WO symptoms, both motor and non motor, increases along with disease duration and has a negative impact on patients QoL.
\end{abstract}

(c) 2013 Elsevier Ltd. All rights reserved.

\footnotetext{
* Corresponding author. IRCCS San Raffaele Pisana di Roma, Dipartimento di Neurologia, Via della Pisana 216, 00163 Rome, Italy. Tel.: +39 (0) 06522524 56; fax: +39 (0) 0666058486 .

E-mail addresses: fabrizio.stocchi@tin.it, deep@medidata.it (F. Stocchi), angelo3000@yahoo.com (A. Antonini), pbarone@unisa.it (P. Barone), michele. tinazzi@ospedaleuniverona.it (M. Tinazzi), m.zappia@unict.it (M. Zappia), onofrj@ unich.it (M. Onofrj), stefano.ruggieri@uniroma1.it (S. Ruggieri), morgante@unime. it (L. Morgante), u.bonuccelli@med.unipi.it (U. Bonuccelli), leonardo.lopiano@ unito.it (L. Lopiano), peter.pramstaller@eurac.edu (P. Pramstaller), alberto. albanese@unicatt.it, albanese@istituto-besta.it (A. Albanese), mahmood.attar@ novartis.com (M. Attar), valeria.posocco@novartis.com (V. Posocco), delia. colombo@novartis.com (D. Colombo), giabbr@unige.it (G. Abbruzzese).
}

\section{Introduction}

For over 40 years, levodopa has been the gold-standard of treatment of $\mathrm{PD}$, and patients unavoidably will require it during the course of the disease. When patients are initially started on levodopa therapy they experience a sustained clinical response throughout the day. As the disease progresses, the therapeutic effect shortens and many patients experience benefit only for a few hours after ingestion of the drug: this step is otherwise known as 
the WO. Despite several studies have been conducted in patients with fluctuations, it is difficult to infer from the available data a clear consensus on the prevalence, timing and characteristics of the WO phenomenon. For many years, doctors considered the WO as a late and even rare phenomenon. On the other hand, it is now widely recognized that the development of WO has a significant impact on patient's neurological disability and QoL [1-4].

The use of several patient self-assessed questionnaires proved to be an effective tool in the diagnosis of WO, showing higher sensitivity than the routine clinical evaluation [3,5,6]. Among them the WOQ-19 has recently been recognized by a task force of the Movement Disorders Society as "recommended diagnostic screening tool in PD" [7].

The current study was based on an observational, cross-sectional, multicentre study called Early DEtection of wEaring off in Parkinson disease (DEEP Study). The primary goal was to look at the frequency of WO phenomena among a wide population of patients with PD, and secondarily to assess the impact of WO on patient's QoL.

\section{Methods}

\subsection{Study design}

The DEEP study included a preliminary phase, recently published [8], that had as objective the linguistic and the psychometric validation as well as the diagnostic capacity assessment of the Italian version of the WOQ-19. In this manuscript we present the results of the core phase of the study, an epidemiological survey on WO symptoms and their associated phenomena, in a population of patients with PD.

From March to October 2010, we have prospectively recruited patients with PD to participate in the DEEP study, conducted at 37 movement disorder clinics in Italy. A total of 634 patients were evaluated, 207 have also participated to the WOQ-19 diagnostic capacity study [8]. The inclusion criteria were as follows: 1 ) age $\geq 18$ years; 2) a diagnosis of PD [9]; 3) levodopa and/or dopamine agonists (DAs) therapy for $\geq 1$ year before the study screening; 4) ability to understand and fill out the WOQ-19. The exclusion criteria included the following: 1) a Mini-Mental State Examination (MMSE) score $\leq 23$ and/or a diagnosis of dementia according to DSM-IV; 2) any severe disabling conditions adversely affecting the patient's mental status; 3) Deep Brain Stimulation treatment; 4) any infusional therapy in the year before the study screening; 5) current inclusion in other clinical trials.

Neurologists experienced in movement disorders collected, following a specific training and during a single visit, standard demographic and clinical information on all participants who met the criteria, via a structured interview and by examination. This included but was not limited to age, sex, educational level,

Table 1

Demographic and clinical data of patients with and without WO, according to the WOQ-19: the DEEP Study.

\begin{tabular}{|c|c|c|c|c|}
\hline & Patients total $(n=617)$ & Patients WOQ-19 $\geq 2(n=415)$ & Patients WOQ $<2(n=202)$ & $p$ value \\
\hline $\mathrm{M}: \mathrm{F}(\mathrm{n})$ & $381: 236$ & $244: 171$ & $137: 65$ & 0.0304 \\
\hline Age, mean $\pm \mathrm{SD}$ (range) & $66.8 \pm 9.2(29.1-88.5)$ & $65.9 \pm 9.1(29.1-87.3)$ & $68.6 \pm 9.3(31.4-88.5)$ & $0.0005^{*}$ \\
\hline Disease duration, mean $\pm \mathrm{SD}$ (range) & $8.0 \pm 4.7(0.9-25.1)$ & $8.97 \pm 4.9(0.9-25.1)$ & $6.21 \pm 3.9(1.0-20.4)$ & $<0.001^{*}$ \\
\hline Disease duration, $\mathrm{n}(\%)$ & & & & $<0.001^{*}$ \\
\hline$<2.5 \mathrm{y}$ & $55(8.9 \%)$ & $23(41.8 \%)$ & $32(58.2 \%)$ & \\
\hline $2.5-5 \mathrm{y}$ & $130(21.1 \%)$ & $71(54.6 \%)$ & $59(45.4 \%)$ & \\
\hline $5-10 y$ & $238(38.6 \%)$ & $165(69.3 \%)$ & $73(30.7 \%)$ & \\
\hline$>10 y$ & $194(31.4 \%)$ & $156(80.4 \%)$ & $38(19.6 \%)$ & \\
\hline H\&Y staging, $n(\%)$ & & & & $<0.0001^{*}$ \\
\hline Stage 1 & $60(9.6 \%)$ & $25(41.7 \%)$ & $35(58.3 \%)$ & \\
\hline Stage 1.5 & $88(14.3 \%)$ & $48(54.5 \%)$ & $40(45.4 \%)$ & \\
\hline Stage 2 & $220(35.7 \%)$ & $137(62.3 \%)$ & $83(37.7 \%)$ & \\
\hline Stage 2.5 & $139(22.5 \%)$ & $110(79.1 \%)$ & $29(20.8 \%)$ & \\
\hline Stage 3 & $88(14.3 \%)$ & $74(84.1 \%)$ & $14(15.9 \%)$ & \\
\hline Stage 4 & $20(3.2 \%)$ & $19(95.0 \%)$ & $1(5.0 \%)$ & \\
\hline Stage 5 & $2(0.3 \%)$ & $2(100 \%)$ & $0(0 \%)$ & \\
\hline UPDRS total score, mean \pm SD (range) & $37.4 \pm 17.5(5.0-111.0)$ & $39.6 \pm 18.1(5.0-111.0)$ & $32.8 \pm 15.4(10.0-81.0)$ & $<0.0001^{*}$ \\
\hline Part I & $2.2 \pm 1.9(0-11)$ & $2.3 \pm 2.1(0-11)$ & $1.9 \pm 1.6(0-6)$ & 0.0087 \\
\hline Part II & $11.2 \pm 6.2(0-35)$ & $12.3 \pm 6.3(0-35)$ & $9.1 \pm 5.2(0-25)$ & $<0.0001^{*}$ \\
\hline Part III & $24.0 \pm 11.7(3-73)$ & $25.1 \pm 12.3(3-73)$ & $21.6 \pm 10.1(6-60)$ & $0.0007^{*}$ \\
\hline $\begin{array}{l}\text { Anti-Parkinson therapy duration } \\
\quad \text { (years), mean } \pm \text { SD (range) }\end{array}$ & $6.6 \pm 4.6(0-25)$ & $7.4 \pm 4.8(1-25)$ & $4.8 \pm 3.7(0-20)$ & $<0.0001^{*}$ \\
\hline $\begin{array}{l}\text { Levodopa therapy duration, (years), } \\
\text { mean } \pm \text { SD (range; } \mathrm{n} \text { ) }\end{array}$ & $3.62 \pm 3.32(0.22-23.39, n=525)$ & $3.70 \pm 3.58(0.22-23.39 ; n=377)$ & $3.42 \pm 2.55(0.47-13.42 ; n=148)$ & 0.3104 \\
\hline $\begin{array}{l}\text { Levodopa daily dosage, }(\mathrm{mg}) \\
\quad \text { mean } \pm \text { SD (range; } \mathrm{n})\end{array}$ & $420.4 \pm 209.3(50-1500 ; n=534)$ & $439.4 \pm 217.0(50-1500 ; n=386)$ & $370.6 \pm 179.3(100-1200 ; n=148)$ & $0.0002^{*}$ \\
\hline Education (years), $\mathrm{n}$ & & & & 0.3199 \\
\hline None & 17 & 8 & 9 & \\
\hline $3-5$ & 205 & 143 & 62 & \\
\hline $6-8$ & 176 & 119 & 57 & \\
\hline $9-13$ & 148 & 95 & 53 & \\
\hline$>13$ & 71 & 50 & 21 & \\
\hline Employment status, n (\%) & & & & 0.0105 \\
\hline employed & 92 (14.9\%) & $65(15.7 \%)$ & $27(13.4 \%)$ & \\
\hline unemployed & $107(17.3 \%)$ & $84(20.2 \%)$ & $23(11.4 \%)$ & \\
\hline retired & $418(67.7 \%)$ & $266(64.1 \%)$ & $202(75.2 \%)$ & \\
\hline Caregiver assistance, $\mathrm{n}(\%)$ & $112(18.2 \%)$ & $92(22.2 \%)$ & $20(9.9 \%)$ & $0.0002^{*}$ \\
\hline Smoking habits, n (\%) & $233(38.5 \%)$ & $154(37.8 \%)$ & 79 (39.9\%) & 0.6250 \\
\hline Alcohol consumption, $\mathrm{n}(\%)$ & $324(54.5 \%)$ & $209(52.6 \%)$ & $115(58.4 \%)$ & 0.1866 \\
\hline Physical activity, n (\%) & $195(31.6 \%)$ & $126(30.4 \%)$ & $69(34.1 \%)$ & 0.6658 \\
\hline MMSE & $28.1 \pm 1.7(24-30)$ & $28.1 \pm 1.6(24-30)$ & $28.1 \pm 1.7(24-30)$ & 0.9507 \\
\hline
\end{tabular}

Abbreviations: H\&Y = Hoehn \& Yahr scale; UPDRS = Unified Parkinson's Disease Rating Scale; MMSE = Mini Mental State Examination.

*Shows statistically significant $p$-values after Bonferroni's correction (critical $p$-value $=0.0026$ ). 
employment status, need for care, life style habits. Specifically disease related data were collected on age at disease onset, disease duration, UPDRS scores, Hoehn \& Yahr scale (H\&Y), current and previous therapy information, concomitant diseases. Cognitive abilities and QoL were respectively investigated with the MMSE and the 8-item Parkinson's Disease Questionnaire (PDQ-8) [10].

\subsection{Diagnosis of $W O$}

The diagnosis of WO was made both on the neurologist evaluation as well as on the basis of the patient self-assessment using the Italian version of the WOQ-19. Neurologists used a trace of structured interview to patients, which investigated the periodic reappearance of symptoms during the day, the temporal relationship with the intake of at least one scheduled dose of medication in a consistent and predictable modality, and the improvement after intake of the drug. The interview was completed at discretion of the neurologists and the final diagnosis based on the overall impression. The WOQ-19 was filled in by participants in conjunction with the visit, but neurologists were instructed to not interfere with the compilation and were not aware of the questionnaire responses. The questionnaire consists of 9 items assessing fluctuations of motor symptoms, including tremor, difficulty in speech, weakness, problems with balance, slowness, reduced dexterity, general stiffness, muscle cramps, difficulty getting out of the chair; and 10 items assessing fluctuations of non-motor symptoms, including anxiety, sweating, mood changes, numbness, panic attacks, cloudy mind/ dullness of thinking, abdominal discomfort, experience hot and cold, pain, aching [8]. For each item, patients were asked to tick whether symptoms were present and whether they improved after the following dose of anti-Parkinson treatment: a cutoff of $\geq 2$ improved symptoms indicated diagnosis of WO [8].

\subsection{Standard protocol approvals, registrations and patient consents}

The study was approved by the Ethical Committees of each participating institution and written informed consent and processing personal data authorization were obtained from each participant.

\subsection{Statistical analysis}

Descriptive analyses were mean and standard deviation (SD), or median and interquartile range (IQR). Comparisons were performed either by analysis of variance and Chi-square or Fisher exact test. The significance threshold was set to 0.05 with Bonferroni's correction in case of multiple comparisons.

In order to identify the variables associated with WO, logistic regression analysis was performed, considering WOQ score $\geq 2$ as outcome of the dependent variable and as regressors: disease duration, gender, age, education, UPDRS part I, II, III scores, treatment duration, smoking habit, need for care (treatment duration and dose were not considered for the model because available for a lower portion of patients). The effect exerted by these variables on QoL was also tested using linear models with PDQ8 as the continuous dependent variable. Project management including data banking, quality control and statistical analysis, was performed by MEDIDATA (Modena, Italy).

\section{Results}

Of the 634 patients screened, 617 (97.3\%) met the inclusion criteria (Table 1); the excluded patients did not differ significantly on demographic and clinical parameters with respect to the study population. Fifty hundred thirty eight $(87.2 \%)$ patients were taking levodopa, 64 (10.4\%) as monotherapy, 465 (75.3\%) were on DAs. Additional medications were as follows: catechol O-methyltransferase (COMT) inhibitors in 211 (34.2\%) subjects, MAO-B inhibitors in 241 (39.1\%), anticholinergics in 22 (3.6\%). Patients receiving psychotropic drugs were 185 (30.0\%). The most frequent concomitant non-neurological disorders were: hypertension in 184 patients (29.8\%), heart diseases in 75 (12.2\%), diabetes in 54 (8.8\%).

\subsection{Diagnosis of WO}

Neurologists identified 351 subjects (56.9\%) with WO symptoms while 415 subjects (67.3\%) were identified by the WOQ-19. The WOQ-19 was more sensitive particularly in subjects with $<2.5$ years disease duration, with $23(41.8 \%)$ patients indicating $\geq 2 \mathrm{WO}$ symptoms compared with 12 (21.8\%) patients identified by neurologists, similarly for subjects with $2.5-5$ years ( $54.6 \%$ vs. $36.2 \%$ ), $5-10$ years (69.3\% vs.60.1\%), and $>10$ years disease duration $(76.8 \%$ vs. $80.4 \%$; Fig. 1). For 489 (79.3\%) patients there was agreement between neurologists and WOQ-19 for the absence in $170(27.6 \%)$ or presence in $319(51.7 \%)$ of WO. For $128(20.8 \%)$ patients there was disagreement; 32 (5.2\%) those in whom WO diagnosis by neurologists was not confirmed by WOQ-19, and 96 (15.6\%) endorsed WO symptoms through WOQ-19, which their neurologists did not diagnose. When considering levodopa therapy duration, among patients under treatment for $\leq 1$ year, 61 (77.2\%) were diagnosed as having WO by WOQ-19 vs. 47 (59.5\%) by neurologists, in subjects treated for $1-2$ years, 96 (75.6\%) vs. 80 (63.0\%), for $2-3$ years 71 (66.4\%) vs. 62 (57.9\%), for $3-5$ years $65(66.3 \%)$ vs. 54 (55.1\%), 84 (73.7\%) vs. 85 (74.6\%) subjects under levodopa treatment for $>5$ years.

Given the well-established reliability and higher sensitivity of WOQ-19 in respect to the clinical evaluation, and considering also the low degree of disagreement among subjects diagnosed by neurologists and not through WOQ-19, from now on we will only focus on the results based on WOQ-19 data. Overall, the mean number of reported symptoms experienced in a normal day was $8.7 \pm 3.8$, of which $5.6 \pm 2.1$ were motor and $3.1 \pm 2.3$ were nonmotor. Taking into account the symptoms reported as usually improving after the following dose of anti-Parkinson medications (otherwise defined as WO symptoms) they were equal to a total of $4.1 \pm 3.8,3.1 \pm 2.6$ were motor and $1.0 \pm 1.6$ were non motor. The frequency of symptoms reported by patients through the WOQ-19 is listed in Table 2.

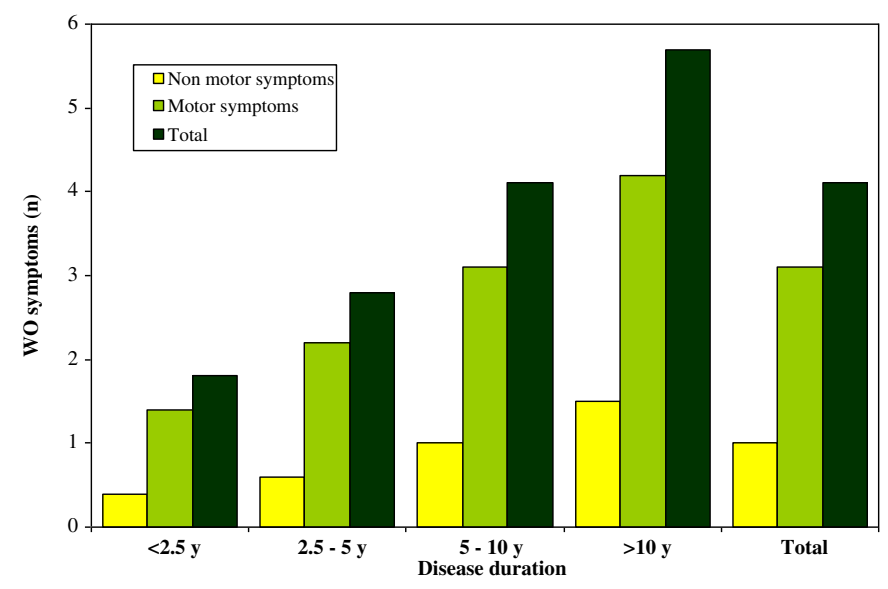

Fig. 1. Mean number of WO symptoms reported through the WOQ-19 by patients with PD, stratified by disease duration: the DEEP study. 


\subsection{Subjects with WO}

Subjects with WO were significantly younger $(65.9 \pm 9.1$ vs. $68.6 \pm 9.3 \mathrm{y} ; p=0.0005)$. Patients with WO had significantly longer disease duration $(8.97 \pm 4.9$ vs. $6.21 \pm 3.9 \mathrm{y} ; p<0.001)$, longer antiParkinson treatment ( $7.4 \pm 4.8$ vs. $4.8 \pm 3.7 \mathrm{y} ; p<0.0001)$, greater disability, as measured by the UPDRS total score (39.6 \pm 18.1 vs. $32.8 \pm 15.4 ; p<0.0001)$, and greater disease severity, as measured by the H\&Y scale ( $p<0.0001$, Table 2 ), compared to subjects without WO. Three hundred and ninety (94.0\%) subjects diagnosed with WO were being treated with levodopa, of whom 246 (84.8\%) in polytherapy, 6 (1.4\%) were treated with DAs in monotherapy and
19 (4.6\%) with DAs in association with MAO-B inhibitors. The duration of treatment with levodopa did not differ between patients with and without WO (3.70 \pm 3.58 vs. $3.42 \pm 2.55$; $p=0.3104$ ), whilst the mean daily levodopa dosage was higher in patients with WO $(439.4 \pm 217.0$ vs. $370.6 \pm 179.3 ; p=0.0002)$.

Regression logistic analysis found age, gender, UPDRS part II score and duration of anti-Parkinson treatment significantly associated with WO. In details, every increase of one year of age was associated with a $5.0 \%$ risk reduction of WO diagnosis $(95 \% \mathrm{CI}$ $0.928-0.973, p<0.0001$ ), while women showed a $80.1 \%$ higher risk of experiencing WO than men (95\% CI 1.134-2.861, $p=0.0126$ ). Moreover, it has been calculated an increased risk of WO,

Table 2

Frequency of experienced symptoms and WO symptoms, as reported by subjects, through the WOQ-19, stratified by disease duration: the DEEP study.

\begin{tabular}{|c|c|c|c|c|c|}
\hline Disease duration & $<2.5$ years $(n=55)$ & $2.5-5.0$ years $(n=130)$ & $5.0-10$ years $(n=238)$ & $>10$ years $(n=194)$ & Total $(n=617)$ \\
\hline \multicolumn{6}{|c|}{ Experienced and WO symptoms, $n(\%)$} \\
\hline \multicolumn{6}{|c|}{ Tremor } \\
\hline Experienced & $36(65.5 \%)$ & $93(71.5 \%)$ & $166(69.7 \%)$ & $131(67.5 \%)$ & $426(69.0 \%)$ \\
\hline WO & $18(32.7 \%)$ & $51(39.2 \%)$ & $119(50.0 \%)$ & $104(53.6 \%)$ & $292(47.3 \%)$ \\
\hline \multicolumn{6}{|l|}{ Difficulty in speech } \\
\hline Experienced & $18(32.7 \%)$ & $47(36.2 \%)$ & $99(41.6 \%)$ & $115(59.3 \%)$ & $279(45.2 \%)$ \\
\hline WO & $7(12.7 \%)$ & $22(16.9 \%)$ & $38(16.0 \%)$ & $66(34.0 \%)$ & $133(21.6 \%)$ \\
\hline \multicolumn{6}{|l|}{ Weakness } \\
\hline Experienced & $29(52.7 \%)$ & $91(70.0 \%)$ & $158(66.4 \%)$ & 155 (79.9\%) & $433(70.2 \%)$ \\
\hline WO & $7(12.7 \%)$ & $34(26.2 \%)$ & $83(34.9 \%)$ & $87(44.8 \%)$ & $211(34.2 \%)$ \\
\hline \multicolumn{6}{|c|}{ Problems with balance } \\
\hline Experienced & $22(40.0 \%)$ & $51(39.2 \%)$ & $119(50.0 \%)$ & $127(65.5 \%)$ & 319 (51.7\%) \\
\hline WO & $4(7.3 \%)$ & $21(16.2 \%)$ & $52(21.8 \%)$ & $60(30.9 \%)$ & 137 (22.2\%) \\
\hline \multicolumn{6}{|c|}{ Slowness of movements } \\
\hline Experienced & $43(78.2 \%)$ & 97 (74.6\%) & $210(88.2 \%)$ & $174(89.7 \%)$ & $524(84.9 \%)$ \\
\hline WO & $16(29.1 \%)$ & $53(40.8 \%)$ & $141(59.2 \%)$ & $134(69.1 \%)$ & $344(55.8 \%)$ \\
\hline \multicolumn{6}{|l|}{ Reduced dexterity } \\
\hline Experienced & $38(69.1 \%)$ & $83(63.8 \%)$ & $192(80.7 \%)$ & $170(87.6 \%)$ & $483(78.3 \%)$ \\
\hline WO & $13(23.6 \%)$ & $42(32.3 \%)$ & $124(52.1 \%)$ & $122(62.9 \%)$ & $301(48.8 \%)$ \\
\hline \multicolumn{6}{|l|}{ General stiffness } \\
\hline Experienced & $15(27.3 \%)$ & $56(43.1 \%)$ & $133(55.9 \%)$ & $133(68.6 \%)$ & 337 (54.6\%) \\
\hline WO & $4(7.3 \%)$ & 35 (26.9\%) & $94(39.5 \%)$ & $108(55.7 \%)$ & $241(39.1 \%)$ \\
\hline \multicolumn{6}{|l|}{ Muscle cramps } \\
\hline Experienced & 27 (49.1\%) & $66(50.8 \%)$ & 127 (53.4\%) & $111(57.2 \%)$ & 331 (53.6\%) \\
\hline WO & $4(7.3 \%)$ & $18(13.8 \%)$ & $39(16.4 \%)$ & $46(23.7 \%)$ & 107 (17.3\%) \\
\hline \multicolumn{6}{|c|}{ Difficulty getting out of chair } \\
\hline Experienced & $18(32.7 \%)$ & $42(32.3 \%)$ & $112(47.1 \%)$ & $123(63.4 \%)$ & 295 (47.8\%) \\
\hline WO & $2(3.6 \%)$ & $15(11.5 \%)$ & $59(24.8 \%)$ & $82(42.3 \%)$ & $158(25.6 \%)$ \\
\hline \multicolumn{6}{|l|}{ Anxiety } \\
\hline Experienced & $26(47.3 \%)$ & $65(50.0 \%)$ & $122(51.3 \%)$ & $113(58.2 \%)$ & $326(52.8 \%)$ \\
\hline WO & $8(14.5 \%)$ & $15(11.5 \%)$ & $46(19.3 \%)$ & $45(23.2 \%)$ & $114(18.5 \%)$ \\
\hline \multicolumn{6}{|l|}{ Sweating } \\
\hline Experienced & $11(20.0 \%)$ & $35(26.9 \%)$ & $83(34.9 \%)$ & $104(53.6 \%)$ & $233(37.8 \%)$ \\
\hline WO & $0(0.0 \%)$ & $7(5.4 \%)$ & $15(6.3 \%)$ & $32(16.5 \%)$ & $54(8.8 \%)$ \\
\hline \multicolumn{6}{|l|}{ Mood changes } \\
\hline Experienced & $22(40.0 \%)$ & $53(40.8 \%)$ & $108(45.4 \%)$ & $98(50.5 \%)$ & $281(45.5 \%)$ \\
\hline WO & $7(12.7 \%)$ & $13(10.0 \%)$ & $46(19.3 \%)$ & $45(23.2 \%)$ & $111(18.0 \%)$ \\
\hline \multicolumn{6}{|l|}{ Numbness } \\
\hline Experienced & $9(16.4 \%)$ & $21(16.2 \%)$ & $52(21.8 \%)$ & $53(27.3 \%)$ & $135(21.9 \%)$ \\
\hline WO & $1(1.8 \%)$ & $3(2.3 \%)$ & $15(6.3 \%)$ & $18(9.3 \%)$ & $37(6.0 \%)$ \\
\hline \multicolumn{6}{|l|}{ Panic attacks } \\
\hline Experienced & $3(5.5 \%)$ & $9(6.9 \%)$ & $28(11.8 \%)$ & $33(17.0 \%)$ & $73(11.8 \%)$ \\
\hline WO & $1(1.8 \%)$ & $1(0.8 \%)$ & $7(2.9 \%)$ & $13(6.7 \%)$ & $22(3.6 \%)$ \\
\hline \multicolumn{6}{|l|}{ Cloudy mind } \\
\hline Experienced & $8(14.5 \%)$ & $31(23.8 \%)$ & $80(33.6 \%)$ & 75 (38.7\%) & $194(31.4 \%)$ \\
\hline WO & $2(3.6 \%)$ & $5(3.8 \%)$ & $29(12.2 \%)$ & $33(17.0 \%)$ & $69(11.2 \%)$ \\
\hline \multicolumn{6}{|c|}{ Abdominal discomfort } \\
\hline Experienced & $13(23.6 \%)$ & $36(27.7 \%)$ & $48(20.2 \%)$ & $53(27.3 \%)$ & $150(24.3 \%)$ \\
\hline WO & $1(1.8 \%)$ & $2(1.5 \%)$ & $11(4.6 \%)$ & $13(6.7 \%)$ & $27(4.4 \%)$ \\
\hline Feelings of hot/col & & & & & \\
\hline Experienced & $6(10.9 \%)$ & $21(16.2 \%)$ & $54(22.7 \%)$ & $50(25.8 \%)$ & $131(21.2 \%)$ \\
\hline WO & $1(1.8 \%)$ & $3(2.3 \%)$ & $11(4.6 \%)$ & $15(7.7 \%)$ & $30(4.9 \%)$ \\
\hline Pain & & & & & \\
\hline Experienced & $12(21.8 \%)$ & $43(33.1 \%)$ & $84(35.3 \%)$ & $64(33.0 \%)$ & $203(32.9 \%)$ \\
\hline WO & $0(0.0 \%)$ & $13(10.0 \%)$ & $21(8.8 \%)$ & $31(16.0 \%)$ & 65 (10.5\%) \\
\hline Aching & & & & & \\
\hline Experienced & $8(14.5 \%)$ & 37 (28.5\%) & $81(34.0 \%)$ & $74(38.1 \%)$ & 200 (32.4\%) \\
\hline WO & $2(3.6 \%)$ & $14(10.8 \%)$ & 30 (12.6\%) & $44(22.7 \%)$ & 90 (14.6\%) \\
\hline
\end{tabular}


respectively equal to $7.9 \%$ for each point increase in UPDRS part II score (95\% CI 1.017-1.144, $p=0.0117)$, and equal to $13.0 \%$ for each additional year of anti-Parkinson treatment duration (95\% CI 1.031-1.245, $p=0.0094)$. The estimated risk for WO diagnosis resulted unmodified even for ever tobacco smokers and alcohol consumers.

\subsection{WO and $\mathrm{QoL}$}

Subjects with WO reported worse Qol, as assessed by PDQ-8, than subjects without WO (32.7 \pm 19.2 vs. $21.4 \pm 15.7$, $p<0.0001)$. The number of motor symptoms $(0.34008 ; p<0.0001)$ as well as non-motor symptoms $(0.33595 ; p<0.0001)$ correlated with PDQ-8 score. By linear regression analysis, the presence of WO symptoms, as identified by WOQ-19, corresponded to an increase of 1.15 points of the PDQ-8 score $(p<0.0001)$. Moreover, disease severity assessed by H\&Y scale $(p=0.0003)$ and the need for caregiver assistance $(p<0.0001)$ also showed a negative impact on patient's QoL.

\section{Discussion}

Our results indicate WO is a potentially "early" phenomenon, as already $41.8 \%$ of the PD patients with $<2.5$ years disease duration reported symptoms according to the WOQ-19 and $21.8 \%$ according to neurologist assessment. The prevalence of WO was higher in subjects with longer disease duration, and patients with $>10$ years of disease duration were diagnosed in $80.4 \%$ and $76.8 \%$ of the cases, respectively by WOQ-19 and by neurologist assessments. As expected, the vast majority of patients with WO were on levodopa (94\%). Interestingly, the levodopa treatment duration did not significantly differ between patients with and without WO and logistic regression analysis revealed that the time since initiation of levodopa did not predict occurrence of WO. Similarly in a recent observational study [12], even if frequency of WO symptoms increased with the total duration of levodopa treatment, the changes were minimal and $86.7 \%$ of patients treated for $<1$ year with levodopa were rated as having WO based on WOQ-9 questionnaire vs. $89.5 \%$ for $<5$ years, and $92.7 \%$ for $>5$ years of levodopa treatment.

This is a marked greater prevalence than previously estimated in the literature. Older studies had showed that about $10 \%$ of patients per year develop motor fluctuations after initiation of treatment with levodopa [13], and within 4-6 years of treatment up to $40 \%$ of patients develop WO [14], while almost all subjects would fluctuate after 10 years $[1,15]$. More recent studies are consisted with ours and have documented that fluctuations of parkinsonian features may occur from the very early stages and WO symptoms can be observed in patients a few months after the initiation of levodopa treatment $[16,17]$. Up to $50 \%$ of patients experience motor fluctuations already by 2 years of treatment [18-20]. Our results confirm the finding of WO in a large percentage of patients with disease duration of $<2.5$ years but clarify that manifestations are predominantly motor symptoms (Table 2 ).

Despite many studies have been conducted in PD patients with fluctuations, these differed in the definition of WO, heterogeneity of study populations, different treatment regimens in accordance with different standard of care that has changed over time. Most of the data are extrapolated from interventional studies that may be biased and probably are not representative of the overall population of patients with PD; in these studies the identification of WO was usually not among the primary objectives and howsoeverdifferent assessment methodologies have been applied. Moreover, the epidemiological data available to date have largely focused on motor fluctuations while non motor symptoms, difficult to assess but no less bothersome, have been often overlooked [3,21-24]. The strengths of our results are the large population-based study, focused to collect data about WO phenomenon and its related manifestations in a large sample of clinically well-characterized patients with $\mathrm{PD}$, in standardized manner, using both clinical assessment and the WOQ-19, which today represents one of the most sensitive methods for WO screening. Assuming WO as transverse phenomenon in the clinical course of PD, we chose an observational design, including subjects under different treatments at all stages of the disease, aiming to photograph the WO phenomenon in a context of the current clinical practice. In addition, our study has allowed to assess the impact of WO symptoms on patient's QoL.

Concerning clinical assessment, the rates of WO diagnosis performed by our neurologists were generally higher compared to other studies [12]. This could be due to heterogeneity of study populations, or more likely because all our examining physicians were movement disorders specialists, who had been specifically trained with the task of completing a well-structured interview focused to identify WO. Greater awareness and experience in collecting data from patient medical history and asking the "right"

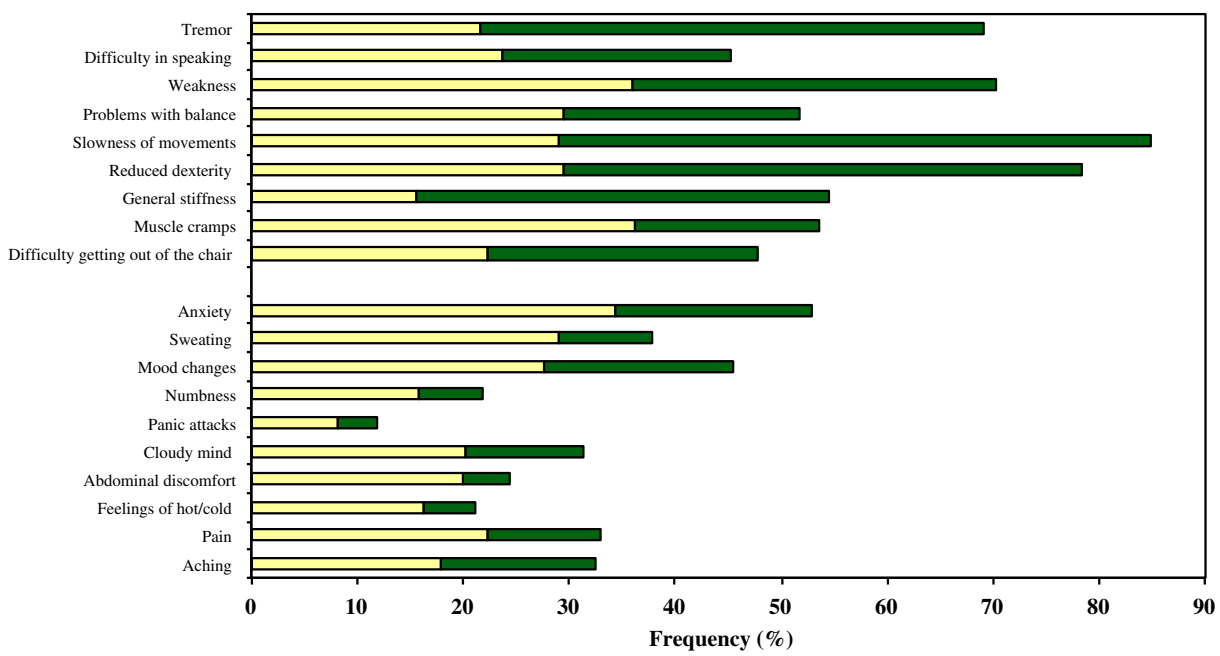

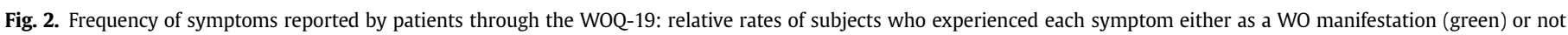
(yellow). 
questions can help make a more timely diagnosis of WO. In addition, patients filled-out the WOQ-19 just before being evaluated by neurologists, and the order of these two diagnostic steps was not randomized. A bias is plausible, as the patient after the completion of the WOQ-19 could be more willing to report symptoms to the neurologist, in this increasing the percentage of diagnosis of WO by the neurologist.

For WO diagnosis through the WOQ-19 we arbitrarily adopted a cut-off of at least two symptoms reported as fluctuating along with medication intake. This cut-off has been identified in the validation study as the best compromise in sensitivity and specificity [8]. In the present study WOQ-19 data were not analysed for different cutoffs, and most likely higher values would be in better agreement with the neurologist's decision. Nevertheless, also our data showed a marked discrepancy in perception between patients and neurologists, which has already been extensively discussed in previous studies $[6,12,25,26]$, and we agree that WO is potentially verifiable at every stage of disease, and it is often underestimated by the routine clinical assessment $[4,12,15,16]$. In our population, almost three out of four WO manifestations were motor symptoms (Table 2, Fig. 2), with slowness of movements (55.8\%) and reduced dexterity (48.8\%) being the most common motor features, anxiety (18.5\%) and mood changes (18.0\%) the most common non motor features. Non-motor symptoms were generally less frequently experienced, in proportion less frequently reported as fluctuating with treatment. The number of WO symptoms, both motor and non motor, increased in accordance with disease duration (Fig. 1, Table 2), and correlated significantly with QoL. Each additional symptom indicated by patients through the WOQ-19 was associated with proportional worsening of the PDQ8 score. We found this result interesting also because highlight that the WOQ-19 could be potentially exploited not only in qualitative (diagnosis of WO), but also in quantitative terms (number of symptoms). Future studies could investigate the correlation between WOQ-19 and other clinical features, in addition to QoL, and eventually validated as measures of follow-up outcome.

In our cohort, patients with WO were significantly younger and the percentage of women was higher than in patients without WO. Every additional year of age was associated with a 5.0\% risk reduction of WO, whilst female gender was associated with an $80.1 \%$ higher risk of WO. These findings are comparable to those observed in a similar study, in which patients with WO were significantly younger and with a higher ratio of women, than those without WO [5]. Conversely, in a British community-based study, younger patients developed dyskinesias sooner than older patients, but there was no correlation with motor fluctuations, moreover men and women did not differ in the occurrence of motor fluctuations or dyskinesias [1]. Thus, in a recent prospective study, female gender was found associated with a shorter time to levodopainduced dyskinesias and younger age at diagnosis was reported as associated with higher likelihood and shorter latency to develop dyskinesias [27].

Our study has some limitations. This is a cross-sectional study and potential risk factors for WO development and prognostic effect of different treatment choices were not assessed. The cohort size of patients with short disease duration is small, perhaps because the broad inclusion criteria have favoured the recruitment of patients at more advanced stages. Therefore for statistical reasons we were forced us to gather data of patients into groups with a relatively wide range of disease duration. Some important clinical variables, such as treatment duration and dose were omitted from the regression model analysis because only partially available. Finally, through the WOQ-19 patients can only report the presence/ absence of fluctuating symptom, but not the degree of severity. Therefore, the information provided by the questionnaire is nevertheless partial and still needs to be integrated with the clinical assessment.

\section{Conclusions}

To our knowledge this is the largest epidemiological study targeting the WO phenomenon as primary objective. Our results indicated WO as potentially observable across all disease stages and that affect patient's QoL. Since WO can be effectively treated by changes in medication dose and kind its recognition is critical given its negative implications on quality of life [28-30].

\section{Acknowledgements}

This study was supported by an educational grant issued by Novartis Pharma S.p.A., Italy. The authors are grateful to Medidata S.r.L. for data collection and analysis and to Shalom Haggiag for helping writing the manuscript.

\section{Appendix 1. The Deep study group}

\begin{tabular}{|c|c|c|c|}
\hline Fabrizio & Stocchi & Dipartimento di Neurologia IRCCS San Raffaele Pisana di Roma & Roma \\
\hline Laura & Vacca & Dipartimento di Neurologia IRCCS San Raffaele Pisana di Roma & Roma \\
\hline Peter P. & Pramstaller & Neurologia Ospedale di Bolzano & Bolzano \\
\hline Maurizio & Facheris & Neurologia Ospedale di Bolzano & Bolzano \\
\hline Mario & Guidotti & Neurologia Ospedale Valduce & Como \\
\hline Elisabetta & Corengia & Neurologia Ospedale Valduce & Como \\
\hline Giulio & Riboldazzi & Neurologia Ospedale di Circolo e Fondazione Macchi & Varese \\
\hline Serena & Leva & Neurologia Ospedale di Circolo e Fondazione Macchi & Varese \\
\hline Alberto & Priori & Neurologia Fondazione Ospedale Maggiore Policlinico & Milano \\
\hline Filippo & Cogiamanian & Neurologia Fondazione Ospedale Maggiore Policlinico & Milano \\
\hline Gianni & Pezzoli & Centro Parkinson Istituti Clinici di Perfezionamento & Milano \\
\hline Canesi & Margherita & Centro Parkinson Istituti Clinici di Perfezionamento & Milano \\
\hline Alberto & Albanese & $\begin{array}{l}\text { Neurologia I Fondazione IRCCS Istituto Nazionale Neurologico C. Besta; } \\
\text { Università Cattolica del Sacro Cuore }\end{array}$ & Milano \\
\hline Paola & Soliveri & Neurologia I Fondazione IRCCS Istituto Nazionale Neurologico C. Besta & Milano \\
\hline Daniele & Picco & Neurologia Riabilitativa Fondazione Salvatore Maugeri IRCCS Veruno & Veruno \\
\hline Fabrizio & Pisano & Neurologia Riabilitativa Fondazione Salvatore Maugeri IRCCS Veruno & Veruno \\
\hline Leonardo & Scarzella & Neurologia Ospedale Evangelico Valdese & Torino \\
\hline Alessia & Tavella & Neurologia Ospedale Evangelico Valdese & Torino \\
\hline Leonardo & Lopiano & Neurologia 4 A.S.O. Molinette & Torino \\
\hline
\end{tabular}


(continued)

\begin{tabular}{|c|c|c|c|}
\hline Maurizio & Zibetti & Neurologia 4 A.S.O. Molinette & Torino \\
\hline Michele & Tinazzi & U.O. Neurologia Ospedale Civile Maggiore - Borgo Trento & Verona \\
\hline Sarah & Ottaviani & U.O. Neurologia Ospedale Civile Maggiore - Borgo Trento & Verona \\
\hline Franco & Valzanìa & Clinica Neurologica Nuovo Ospedale Sant'Agostino-Estense & Modena \\
\hline Sara & Contardi & Clinica Neurologica Nuovo Ospedale Sant'Agostino-Estense & Modena \\
\hline Rocco & Quatrale & UO Neurologia Arcispedale Sant'Anna & Ferrara \\
\hline Mariachiara & Sensi & UO Neurologia Arcispedale Sant'Anna & Ferrara \\
\hline Roberto & Ceravolo & U.O. Neurologia Ospedale Santa Chiara & Pisa \\
\hline Carlo & Rossi & U.O. Neurologia Ospedale Santa Chiara & Pisa \\
\hline Massimo & Cincotta & Neurologia Azienda Sanitaria Firenze - Piero Palagi & Firenze \\
\hline Paola & Vanni & Neurologia Azienda Sanitaria Firenze - Piero Palagi & Firenze \\
\hline Ubaldo & Bonuccelli & U.O. Neurologia Ospedale Versilia & Camaiore \\
\hline Paolo & Del Dotto & U.O. Neurologia Ospedale Versilia & Camaiore \\
\hline Maria Gabriella & Ceravolo & Clinica Neuroriabilitazione Az. Ospedali Riuniti & Ancona \\
\hline Marianna & Capecci & Clinica Neuroriabilitazione Az. Ospedali Riuniti & Ancona \\
\hline Roberta & Marchese & Centro Parkinson - Dipartimento Neuroscienze Università degli Studi di Genova & Genova \\
\hline Tiziano & Tamburini & Centro Parkinson - Dipartimento Neuroscienze Università degli Studi di Genova & Genova \\
\hline Astrid & Thomas & CeSI-Centro Studi Invecchiamento Fondazione Università Gabriele D’Annunzio & Chieti \\
\hline Iole & Borrelli & CeSI-Centro Studi Invecchiamento Fondazione Università Gabriele D'Annunzio & Chieti \\
\hline Roberto & Marconi & Neurologia Ospedale della Misericordia & Grosseto \\
\hline Lucia & Grasso & Neurologia Ospedale della Misericordia & Grosseto \\
\hline Paolo & Stanzione & Clinica Neurologica Policlinico Tor Vergata & Roma \\
\hline Valerio & Pisani & Clinica Neurologica Policlinico Tor Vergata & Roma \\
\hline Anna Rita & Bentivoglio & Neurologia Università Cattolica S. Cuore Policlinico Gemelli & Roma \\
\hline Giovanna & Lorìa & Neurologia Università Cattolica S. Cuore Policlinico Gemelli & Roma \\
\hline Maria Francesca & De Pandis & U.O. Riabilitazione Parkinson Ospedale San Raffaele Cassino & Cassino \\
\hline Giovanna & Federici & U.O. Riabilitazione Parkinson Ospedale San Raffaele Cassino & Cassino \\
\hline Valentino & Manzo & Neurologia, Padiglione F,Amb UVA AORN A. Cardarelli & Napoli \\
\hline Alfonso & Mauro & Struttura Dipartimentale Neuropsicologia AORN San Giovanni di Dio e Ruggi di Aragona & Salerno \\
\hline Paolo & Barone & Centro Parkinson Dipartimento Scienze Neurologiche Università Federico II Napoli & Napoli \\
\hline Marina & Picillo & Centro Parkinson Dipartimento Scienze Neurologiche Università Federico II Napoli & Napoli \\
\hline Stefano & Ruggieri & Neurologia Istituto Mediterraneo Neuromed & Pozzilli \\
\hline Nicola & Modugno & Neurologia Istituto Mediterraneo Neuromed & Pozzilli \\
\hline Paolo & Lamberti & Neurologia “Amaducci” Az. Osp. Univ. Policlinico Consorziale & Bari \\
\hline Claudia & Dell'Aquila & Neurologia “Amaducci” Az. Osp. Univ. Policlinico Consorziale & Bari \\
\hline Giulio & Cicarelli & Neurologia A.O.R.N. San Giuseppe Moscati & Avellino \\
\hline Aldo & Quattrone & Clinica Neurologica Università Magna Grecia & Catanzaro \\
\hline Giuseppe & Nicoletti & Clinica Neurologica Università Magna Grecia & Catanzaro \\
\hline Antonino & Cannas & Neurologia Policlinico Universitario di Monserrato & Monserrato \\
\hline Paolo & Solla & Neurologia Policlinico Universitario di Monserrato & Monserrato \\
\hline Mario & Zàppia & Clinica Neurologica I Policlinico Universitario & Catania \\
\hline Alessandra & Nicoletti & Clinica Neurologica I Policlinico Universitario & Catania \\
\hline Letterio & Morgante & Clinica Neurologica Policlinico G. Martino & Messina \\
\hline Francesca & Morgante & Clinica Neurologica Policlinico G. Martino & Messina \\
\hline Marco & D'Amelio & Neurologia Az. Osp. Univ. Policlinico “P. Giaccone” & Palermo \\
\hline Valeria & Terruso & Neurologia Az. Osp. Univ. Policlinico “P. Giaccone” & Palermo \\
\hline Roberto & Eleopra & SOC Neurologia Az. Osp. Univ. S.Maria della Misericordia & Udine \\
\hline Marco & Mucchiut & SOC Neurologia Az. Osp. Univ. S.Maria della Misericordia & Udine \\
\hline Manuela & Pilleri & U.O. Malattia di Parkinson IRCCS Ospedale San Camillo & Venezia \\
\hline Roberta & Biundo & U.O. Malattia di Parkinson IRCCS Ospedale San Camillo & Venezia \\
\hline Stefania & Nassetti & U.O.C. Neurologia Ospedale Bellaria & Bologna \\
\hline Roberto & Michelucci & U.O.C. Neurologia Ospedale Bellaria & Bologna \\
\hline
\end{tabular}

\section{References}

[1] Schrag A, Quinn N. Dyskinesias and motor fluctuations in Parkinson's disease. A community-based study. Brain 2000;123:2297-305.

[2] Sethi KD. The impact of levodopa on quality of life in patients with Parkinson disease. Neurologist 2010;16:76-83.

[3] Stacy M, Bowron A, Guttman M, Hauser R, Hughes K, Larsen JP, et al. Identification of motor and nonmotor wearing-off in Parkinson's disease: comparison of a patient questionnaire versus a clinician assessment. Mov Disord 2005;20:726-33.

[4] Stacy M. The wearing-off phenomenon and the use of questionnaires to facilitate its recognition in Parkinson's disease. J Neural Transm 2010;117:837-46.

[5] Silburn PA, Mellick GD, Vieira BI, Danta G, Boyle RS, Herawati L. Utility of a patient survey in identifying fluctuations in early stage Parkinson's disease. J Clin Neurosci 2008;15:1235-9.

[6] Martinez-Martin P, Tolosa E, Hernandez B, Badia X. ValidQUICK Study Group. Validation of the "OUICK" questionnaire-a tool for diagnosis of "wearing-off" in patients with Parkinson's disease. Mov Disord 2008;23:830-6.

[7] Antonini A, Martinez-Martin P, Chaudhuri RK, Merello M, Hauser R, Katzenschlager R, et al. Wearing-off scales in Parkinson's disease: critique and recommendations. Mov Disord 2011;26:2169-75.

[8] Abbruzzese G, Antonini A, Barone P, Stocchi F, Tamburini T, Bernardi L, et al. Linguistic, psychometric validation and diagnostic ability assessment of an italian version of a 19-item wearing-off questionnaire for wearing-off detection in Parkinson's disease. Neurol Sci 2012;33:1319-27.

[9] Gelb DJ, Oliver E, Gilman S. Diagnostic criteria for Parkinson disease. Arch Neurol 1999;56:33-9.

[10] Jenkinson C, Fitzpatrick R, Peto V, Greenhall R, Hayman N. The PDQ-8: development and validation of a short-form Parkinson's Disease Questionnaire. Psychol Health 1997;12:805-14.

[12] Bareš M, Rektorová I, Jech R, Farníková K, Roth J, Růžička E, et al. Does WOQ-9 help to recognize symptoms of non-motor wearing-off in Parkinson's disease? J Neural Transm 2012;119:373-80.

[13] Marsden CD, Parkes JD. Success and problems of long-term levodopa therapy in Parkinson's disease. Lancet 1977;1:345-9.

[14] Ahlskog JE, Muenter MD. Frequency of levodopa-related dyskinesias and motor fluctuations as estimated from the cumulative literature. Mov Disord 2001;16:448-58.

[15] Parkinson Study Group. Impact of deprenyl and tocopherol treatment on Parkinson's disease in DATATOP patients requiring levodopa. Ann Neurol 1996;39:37-45.

[16] Fahn S, Oakes D, Shoulson I, Kieburtz K, Rudolph A, Lang A, et al. Levodopa and the progression of Parkinson's disease. N Engl J Med 2004;351:2498508.

[17] the Parkinson Study Group. Does levodopa slow or hasten the rate of progression of Parkinson's disease? J Neurol 2005;252:IV37-42. 
[18] Parkinson Study Group. Pramipexole vs levodopa as initial treatment for Parkinson disease: a randomized controlled trial. Parkinson Study Group. J Am Med Assoc 2000;284:1931-8.

[19] Sato K, Hatano T, Yamashiro K, Kagohashi M, Nishioka K, Izawa N, et al. Prognosis of Parkinson's disease: time to stage III, IV, V, and to motor fluctuations. Mov Disord 2006;21:1384-95.

[20] Stocchi F. Prevention and treatment of motor fluctuations. Parkinsonism Relat Disord 2003;9:S73-81.

[21] Adler CH. Relevance of motor complications in Parkinson's disease. Neurology 2002;58:S51-6.

[22] Witjas T, Kaphan E, Azulay JP, Blin O, Ceccaldi M, Pouget J, et al. Nonmotor fluctuations in Parkinson's disease: frequent and disabling. Neurology 2002;59:408-13.

[23] Politis M, Wu K, Molloy S, G Bain P, Chaudhuri KR, Piccini P. Parkinson's disease symptoms: the patient's perspective. Mov Disord 2010;25:1646-51.

[24] Weintraub D, Moberg PJ, Duda JE, Katz IR, Stern MB. Effect of psychiatric and other nonmotor symptoms on disability in Parkinson's disease. J Am Geriatr Soc 2004;52:784-8.

[25] Stacy MA, Murphy JM, Greeley DR, Stewart RM, Murck H, Meng X, et al. The sensitivity and specificity of the 9-item wearing-off Questionnaire. Parkinsonism Relat Disord 2008;14:205-12.
[26] Melo LM, Chien HF, Barbosa ER. Identification of wearing-off manifestations (reduction of levodopa effect) in Parkinson's disease using specific questionnaire and comparison of the results with routine ambulatory evaluations. Arq Neuropsiquiatr 2010;68:506-10.

[27] Hassin-Baer S, Molchadski I, Cohen OS, Nitzan Z, Efrati L, Tunkel O, et al. Gender effect on time to levodopa-induced dyskinesias. J Neurol 2011;258: $2048-53$.

[28] Nissinen H, Kuoppamäki M, Leinonen M, Schapira AH. Early versus delayed initiation of entacapone in levodopa-treated patients with Parkinson's disease: a long-term, retrospective analysis. Eur J Neurol 2009;16: 1305-11.

[29] Fung VS, Herawati L, Wan Y. Movement disorder Society of Australia clinical research and trials group; QUEST-AP Study Group. Quality of life in early Parkinson's disease treated with levodopa/carbidopa/entacapone. Mov Disord 2009;24:25-31.

[30] Antonini A, Chaudhuri KR, Martinez-Martin P, Odin P. Oral and infusion levodopa-based strategies for managing motor complications in patients with Parkinson's disease. CNS Drugs 2010;24:119-29. 